\title{
Germinal vesicle position and meiotic maturation in mouse oocyte
}

\author{
Stéphane Brunet" and Bernard Maro ${ }^{1,2}$ \\ ${ }^{1}$ UMR 7622 Biologie du Développement, CNRS-UPMC, 9 quai St Bernard, 75005 Paris, France and ${ }^{2}$ Sackler Faculty \\ of Medicine, Tel Aviv University, Ramat Aviv 69978, Israel
}

Correspondence should be addressed to S Brunet; Email: sbrunet@snv.jussieu.fr

\begin{abstract}
During meiotic maturation, mammalian oocytes undergo an asymmetric division which is crucial for the formation of a functional gamete. In various organisms, accurate positioning of the nucleus before $\mathrm{M}$-phase plays a major role in asymmetric cell divisions. However, the role of the position of the nucleus (or germinal vesicle, GV) during the prophase I arrest has not been investigated in mammalian oocytes. Here, we show that incompetent mouse oocytes possess a peripheral GV, while competent oocytes mainly exhibit a central position of the GV. At that time, the position of the GV correlates with the ability of the oocyte to complete meiotic maturation. Moreover, a lower efficiency in GV centering and a reduced ability to progress through meiosis are observed in oocytes from old mice. Thus, the position of the GV could be used as a simple morphological marker of oocyte quality.

Reproduction (2007) 133 1069-1072
\end{abstract}

In mammals, oocytes are kept arrested for an exceptionally extended period during prophase I of meiosis. At that stage, the nucleus is called the germinal vesicle (GV). Upon a surge of luteinizing hormone, they can enter meiotic maturation and undergo the first meiotic division whose accuracy is essential to prevent aneuploidy, which is deleterious for the embryo (Hassold \& Hunt 2001). Alterations of this division increase with maternal age and account for a significant decrease in the production of normal gametes (Ottolenghi et al. 2004). The meiotic divisions are asymmetric and generate a tiny polar body and a large oocyte, which contains most of the cytoplasmic stores and organelles. Asymmetry, which becomes altered with aging (Diaz \& Esponda 2004), is based on the migration of the spindle during meiosis I from the position previously occupied by the GV to the closest domain of the oocyte cortex (Verlhac et al. 2000). The accurate positioning of the nucleus has been shown to be a key event for asymmetric cell divisions in various systems (Gonczy 2002), but very little is known about this process in mammalian oocytes. Nevertheless, since the position of the GV defines the starting point for spindle migration in the oocyte, it may in turn influence the success of the first meiotic division and the formation of a functional gamete. The position of the GV has been documented in mammalian species, but some conclusions remain unclear or debated due the scarcity of the experimental material (Albertini \& Barrett 2004). In addition, the potential impact of this parameter on the completion of meiotic maturation, as well as the relation with maternal age, has not been so far investigated. Thus, we decided to study these questions in the mouse oocyte.

The ovaries were removed from OF1 female mice (Charles River Laboratories, Boston, MA, USA) and transferred to pre-warmed medium. The ovary surface was scarified to allow a rapid release of the oocytes enclosed in antral follicles. The oocytes were denuded and put in culture as previously described (Brunet et al. 2003). Oocytes were carefully positioned in the culture chamber to visualize the GV at its most peripheral position. Transmitted light pictures (Fig. 1) were rapidly taken using $20 \times$ NA0.5 objective lenses (Leica) and a CCD camera (Micromax, Roper Scientific, Tucson, AZ, USA) under a video microscope (Leica DM IRBE) enclosed in a thermostatic chamber (Life Imaging Services Reinach, Switzerland). From the original pictures, GV and oocyte boundaries were outlined (Fig. 1) using Adobe Photoshop CS (Adobe Systems Inc.) and the corresponding binarized images were generated. Image (NIH) was used for image quantitative analysis. For each oocyte and its corresponding GV, the area $(A)$, centroid ( $C_{\mathrm{OO}}$ and $\left.C_{\mathrm{GV}}\right)$, and Feret's radius ( $R_{\mathrm{OO}}$ and $\left.R_{\mathrm{GV}}\right)$ were calculated. The area corresponds to the surface of the projection of the oocyte on a plane. The centroid represents the center point of the selected object and the Feret's radius represents half of the longest distance between any two points of the selected object 
A

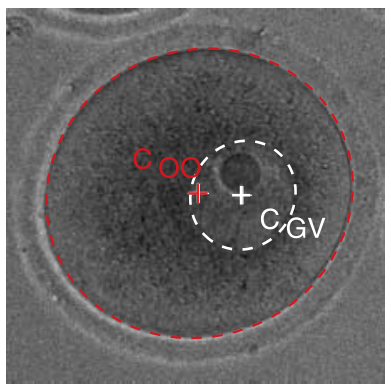

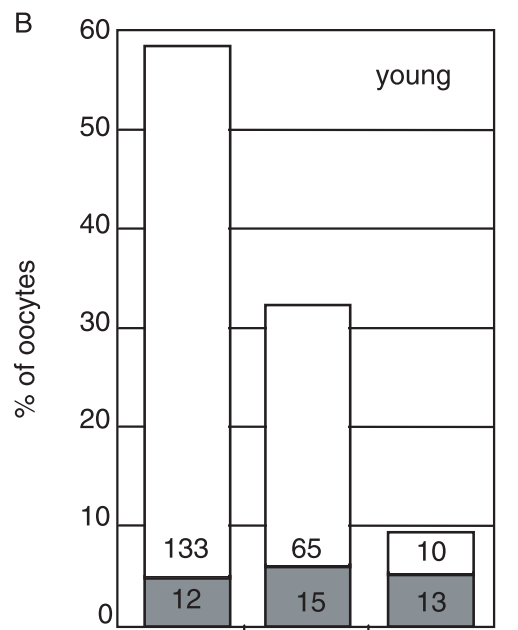

$\rho:[0-0.33][0.33-0.66][0.66-1]$

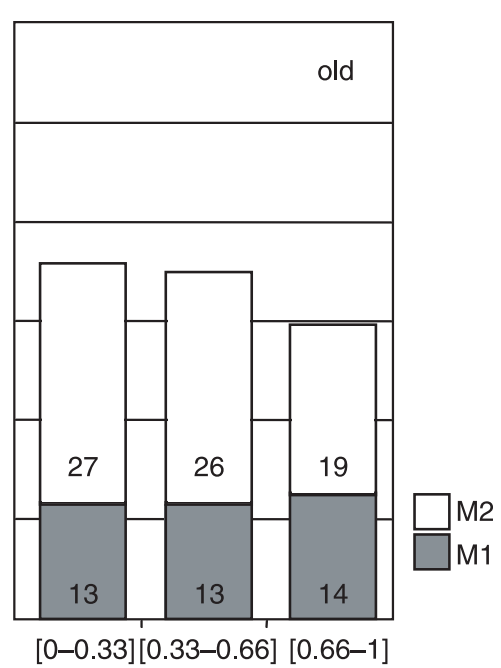

Figure 1 Quantification of the position of the GV and the ability to achieve meiotic maturation of oocytes collected from young or old mice. (A) Transmitted light picture of a mouse oocyte. GV and oocyte boundaries are outlined in white and red respectively. Oocyte's and GV's centroids ( $C_{\mathrm{OO}}$ and $C_{\mathrm{GV}}$ ) and Feret's radius were determined to calculate $\rho$. (B) Percentages of oocytes extruding or not a polar body upon a culture of $14 \mathrm{~h}$ according to the $\rho$ value. Population of oocytes that extruded their polar body (MII) or remained blocked in meiosis I (MI) are represented in white and gray respectively. The number of oocytes scored is indicated within the bar. boundary. Using these values, the parameter $\rho$ was calculated for each oocyte as follows:

$\rho=\frac{\mathrm{d}\left(C_{\mathrm{GV}}-C_{\mathrm{OO}}\right)}{\left(R_{\mathrm{OO}}-R_{\mathrm{GV}}\right)}$

$\rho$ is the ratio between the distance separating the centroids of the oocyte and its GV and the difference between oocyte Feret's radius and the GV Feret's radius. This parameter defines the absolute position of the GV within the oocyte. In theory, it varies from 0 to 1 respectively when the GV is at the perfect center of the oocyte and when it contacts the cortex of the oocyte.

To study the position of the GV and its potential influence on meiotic maturation, we first analyzed oocytes that were able to resume meiosis and undergo GV breakdown upon culture. Oocytes were collected from 7- to 11-week-old female OF1 mice, referred as young mice in the text $(n=5)$. They exhibited an average $\rho$ value of $0.32 \pm 0.23$ (Table 1). A $\rho$ value lower than 0.33 is indicative of oocytes with a central GV. In contrast, a $\rho$ value over 0.66 indicates oocytes with a peripheral GV. In between, the GV is at an intermediate position. According to this criterion, we found that more than $58 \%$ of the oocytes collected from young mice had a central GV, while $32 \%$ of the GVs were located in the middle area and only $9 \%$ were peripheral (Fig. 1).

The oocytes were then scored for the extrusion of the first polar body after $14 \mathrm{~h}$ of culture. About $84 \%$ of the oocytes

Table 1 Effect of maternal age on the position of the germinal vesicle (GV) and the ability to achieve meiotic maturation of mouse oocyte.

\begin{tabular}{|c|c|c|c|c|c|c|}
\hline & \multirow[b]{2}{*}{$n$} & \multicolumn{2}{|c|}{ Young } & \multirow[b]{2}{*}{$n$} & \multicolumn{2}{|c|}{ Old } \\
\hline & & $\rho$ & $A$ & & $\rho$ & $A$ \\
\hline GV & 59 & $0.70 \pm 0.15$ & $7168 \pm 916$ & 16 & $0.41 \pm 0.25$ & $9435 \pm 583$ \\
\hline GVBD & 248 & $0.32 \pm 0.23$ & $10383 \pm 960$ & 112 & $0.48 \pm 0.27$ & $10778 \pm 1186$ \\
\hline MI & 40 & $0.51 \pm 0.28$ & $10340 \pm 964$ & 40 & $0.52 \pm 0.28$ & $10638 \pm 1244$ \\
\hline MII & 208 & $0.29 \pm 0.20$ & $10391 \pm 961$ & 72 & $0.46 \pm 0.26$ & $10855 \pm 1154$ \\
\hline
\end{tabular}

Oocytes were collected from 7- to 11-week-old (young) or 1-year-old (old) female mice. Incompetent oocytes (GV), oocytes that entered meiotic maturation (GVBD) and among them, oocytes that remained blocked in meiosis I (MI) or that extruded a polar body (MII) were scored. For each category, the number of oocytes $(n)$, the means \pm s.D. for $(\rho)$ and the area ( $A$ in pixels) are presented. 
extruded the first polar body and arrested at metaphase II. However, when we analyzed the rate of polar body extrusion as a function of $\rho$ (Fig. 1), we observed that $92 \%$ of the oocytes with a central GV $(\rho<0.33)$ did extrude a polar body, while there were $76 \%$ when the GV was intermediate $(0.33<\rho<0.66)$ and only $43 \%$ when it was peripheral $(\rho>0.66)$. The distribution of meiosis I versus metaphase II oocytes in the three populations was significantly different $(P<0.05$, Fisher's test). Thus, in young mice, the ability of the oocyte to achieve meiotic maturation is correlated with the position of the GV and decreases with the distance of the GV from the oocyte center.

To determine a potential effect of maternal age on the position of the GV, oocytes collected from 1-year-old OF1 mice $(n=12)$, referred to as old mice, were studied. As mentioned previously, we analyzed oocytes that were able to resume meiosis. They were significantly bigger than the one collected from young mice ( $P=0.002$, Student's $t$-test, Table 1). Nevertheless, the difference only represents a 3\% increase of the measured area which may reflect a minor variation in the levels of cytoplasmic stores. The oocytes able to undergo GV breakdown upon culture exhibited an average $\rho$ value of $0.48 \pm 0.27$ (Table 1 ). This value is significantly higher than the one measured in young females ( $P<0.001$, Student's $t$-test). The distribution of the oocytes according to $\rho$ indicated that only $34 \%$ of them had a central GV, 30\% a peripheral one, and 35\% an intermediate one. This distribution along a radial axis was homogenous $\left(P=0.923, \chi^{2}\right.$-test $)$ and we wondered whether it was indicative of a random positioning of the $\mathrm{GV}$. If the GV is randomly positioned in the volume of the oocyte, the GV centroid is randomly located on the area representing the projection of the oocyte on a plane. In this case, like on a dartboard, the probability of observing the $\mathrm{GV}$ at a given place increases with the distance from the centroid of the oocyte. As a consequence, the size of the populations of oocytes should increase with the $\rho$ value. Therefore, the distribution we observed did not fit with a random positioning but indicated that the GV of oocytes in old mice kept a tendency to be centered. However, the efficiency in positioning the GV at the center of the oocyte dramatically decreases with maternal age.

The oocytes collected from old mice were then cultured for $14 \mathrm{~h}$ and scored for the extrusion of the polar body. As expected, the rate of polar body extrusion was notably lower than in young females: only $64 \%$ of the oocytes that resumed meiosis extruded a polar body. It has to be noted that the size of oocytes which reached metaphase II was not significantly different from the ones blocked in meiosis $\mathrm{I}(P=0.367$ in the case of old mice and $P=0.76$ in the case of young mice, Student's $t$-test). The rate of polar body extrusion was analyzed as a function of $\rho$ (Fig. 1): about $67 \%$ of the oocytes did extrude their polar body in the first two groups $(\rho<0.33$ and $0.33>\rho>0.66)$ and $58 \%$ in the last group $(\rho>0.66)$. The difference is non-significant (Fisher's $t$-test). In addition, the distribution of meiosis
I- versus metaphase II-arrested oocytes were similar in old mice and in the subpopulation of oocytes collected in young mice and presenting a peripheral GV $(P=0.009$, Fisher's test). These data indicate that the aging-dependent decrease of the ability to achieve meiotic maturation associates with a loss of correlation between this parameter and the position of the GV in the oocyte.

The oocytes unable to resume meiosis were finally analyzed. In old females, only few were recovered. In old and young females, the incompetent oocytes were significantly smaller than the competent ones $(P<0.001$, Student's $t$-test, in young and old females, Table 1), suggesting that they are not fully grown despite being enclosed in antral follicles. In young females, in contrast to competent oocytes, they exhibited a high average $\rho$ value of $0.70 \pm 0.15$. About $66 \%$ of them possessed a peripheral GV $(\rho>0.66)$ and none was found with a central one $(\rho<0.33)$. In order to analyze oocytes at an earlier step of their growth phase, large preantral follicles were recovered from ovaries of young mice. These follicles were dissociated by incubation in pronase (Brunet et al. 1998) to recover the enclosed oocytes. These oocytes $(n=12)$ were small $(A=5708 \pm 630)$ and had an average $\rho$ value of $0.69 \pm 0.14$, indicative of a peripheral GV. These observations indicate that the final steps of oocyte growth and the acquisition of the competence to resume meiosis are associated with a change of position of the GV from the periphery to the center of the oocyte.

We have defined a parameter $\rho$, using quantitative image analysis, to accurately quantify the position of the GV within the oocyte. We found that the position of the GV is regulated in mouse oocytes. In young females, incompetent oocytes are characterized by a peripheral GV. Once the oocytes are fully grown and able to resume meiosis, the $\mathrm{GV}$ becomes central. The position of the GV is at that time correlated with the ability of the oocyte to achieve meiotic maturation: a peripheral GV is unfavorable to polar body extrusion. In addition, the accurate positioning of the GV is affected by maternal age. In old females, the centering efficiency of the GV in competent oocytes is severely reduced and the ability to achieve meiotic maturation does not correlate with the position of the GV anymore. Nevertheless, a general decrease in completion of meiotic maturation is observed. Taken together, these observations suggest that the position of the GV may be used as a simple morphological marker of oocyte quality.

The mechanisms involved in the positioning of the GV during the extended prophase I arrest in mammalian oocytes remain uncharacterized. They most probably involve factor(s) controlling the organization of the oocyte cytoskeleton, as shown in other organisms (Huynh \& St Johnston 2004). In the mouse oocyte, similar factor(s) could generate centripetal forces on the GV and determine its central position. The robustness of this system would decline with maternal age. Interestingly, an age-related weakening of the spindle checkpoint activity, a system that prevents chromosome segregation errors, 
has been documented in mammalian oocytes. It results from the reduction in the levels of transcripts and proteins of some of the components of the spindle checkpoint machinery (for review, see Homer et al. 2005).

Based on our observations, we can hypothesize that a common activity is involved in GV positioning and efficient progression through meiotic maturation. The most likely candidates would be proteins required for the organization of the cytoskeleton during both interphase and M-phase. Alternatively, independent factors could be involved in the different processes and their activities may be similarly reduced with aging. We are currently trying to characterize the mechanisms and factors at play, in order to better understand both the role of GV positioning and the effects of aging on meiotic maturation.

\section{Acknowledgements}

We thank R Schwartzmann and V Georget from the Service $d^{\prime}$ Imagerie of the IFR 83 and MH Verlhac for the critical reading of the manuscript. The authors declare that there is no conflict of interest that would prejudice the impartiality of this scientific work.

\section{References}

Albertini DF \& Barrett SL 2004 The developmental origins of mammalian oocyte polarity. Seminars in Cell and Developmental Biology 15 599-606.
Brunet S, Polanski Z, Verlhac MH, Kubiak JZ \& Maro B 1998 Bipolar meiotic spindle formation without chromatin. Current Biology 8 1231-1234.

Brunet S, Pahlavan G, Taylor S \& Maro B 2003 Functionality of the spindle checkpoint during the first meiotic division of mammalian oocytes. Reproduction 126 443-450.

Diaz H \& Esponda P 2004 Ageing-induced changes in the cortical granules of mouse eggs. Zygote 12 95-103.

Gonczy P 2002 Mechanisms of spindle positioning: focus on flies and worms. Trends in Cell Biology 12 332-339.

Hassold T \& Hunt P 2001 To err (meiotically) is human: the genesis of human aneuploidy. Nature Reviews. Genetics 2 280-291.

Homer HA, McDougall A, Levasseur M \& Herbert M 2005 Restaging the spindle assembly checkpoint in female mammalian meiosis I. Cell Cycle 4 650-653.

Huynh JR \& St Johnston D 2004 The origin of asymmetry: early polarisation of the Drosophila germline cyst and oocyte. Current Biology 14 R438-R449.

Ottolenghi C, Uda M, Hamatani T, Crisponi L, Garcia JE, Ko M, Pilia G, Sforza C, Schlessinger D \& Forabosco A 2004 Aging of oocyte, ovary, and human reproduction. Annals of the New York Academy of Sciences 1034 117-131.

Verlhac MH, Lefebvre C, Guillaud P, Rassinier P \& Maro B 2000 Asymmetric division in mouse oocytes: with or without Mos. Current Biology 10 1303-1306.

Received 22 January 2007

First decision 6 March 2007

Accepted 2 April 2007 